\title{
Personality and Metamotivational Predictors of Aggressive Driving
}

\author{
Kathryn D. Lafreniere, Chris C. Lee, Joan Craig, Dhwani Shah, Kenneth M. Cramer \\ University of Windsor
}

\begin{abstract}
Aggressive driving is an all-too common occurrence and a major cause of serious motor vehicle accidents. This investigation examined relations among individuals' personality and metamotivational tendencies and their propensity for aggressive driving. In Phase 1 of this research, 270 participants recruited from the psychology participant pool of a medium-sized Canadian university completed an online survey that examined propensity for aggressive driving in relation to driving behaviors and measures of personality and motivation, including the Motivational Style Profile (Apter et al., 1998). Phase 2 was a pilot study of 12 participants who took part in a driving simulator experimental session in which participants' reactions to driving scenarios that are intended to elicit aggressive driving were observed and recorded, and pre- and posttest measures of physiological indicators and mood states were administered. Findings from Phase 1 indicated that individuals who were higher in narcissism, impulsivity, negativism, and paratelic dominance showed greater propensity for aggressive driving. In addition, individuals who were high in negativism reported less consistent use of seatbelts, and more negative driving incidents such as previous accidents and incidents of inattentive driving. Negativism was also found to be significantly associated with propensity for aggressive driving in the Phase 2 pilot study, and negativist dominant individuals were found to show decreases in negative mood state and physiological arousal after the simulated driving, relative to conformist dominant participants. Implications of considering metamotivational predictors of aggressive driving are discussed.
\end{abstract}

Keywords: aggressive driving, negativism, risk-taking, driving simulator

Aggressive driving occurs when drivers intentionally harm other drivers within a driving environment (Hennessey \& Wiesenthal, 2002) and is a major cause of vehicle crashes on roadways (McTish \& Park, 2016). Aggressive driving encompasses a number of dysfunctional driving behaviors, including speeding, running red lights, street racing, excessively honking the horn, following too closely, erratic lane changing, failing to yield the right of way, not obeying traffic signs, and passing where prohibited (Traffic Injury Re-

Editorial Board Invited Paper from the 2019 International Reversal Theory Conference, Las Vegas, New Mexico

This research was supported by internal research grants from the Faculty of Arts, Humanities, and Social Sciences and the Faculty of Engineering at the University of Windsor, Canada.

Kathryn D. Lafreniere, Department of Psychology; Chris C. Lee, Department of Civil and Environmental Engineering; Joan Craig, Department of Psychology; Dhwani Shah, Department of Civil and Environmental Engineering; Kenneth M. Cramer, Department of Psychology.

Correspondence concerning this article should be addressed to Kathryn D. Lafreniere. E-mail:lafren1@uwindsor.ca search Foundation, n.d.). According to a 2014 survey of 2,705 licensed drivers in the United States, 78\% of Americans reported engaging in at least one incident of aggressive driving in the past year. It is believed that this is likely an underestimate of the true extent of aggressive driving, since drivers may underreport problematic driving behaviors due to their negative social connotation (AAA Foundation for Traffic Safety, 2016). The present investigation examined personality and motivational predictors of aggressive driving using both an online survey and a pilot study of simulated driving behavior, with a particular focus on reversal theory's metamotivational states.

Several previous investigations have examined personality and motivational factors in relation to aggressive driving, but most of these studies have employed correlational techniques to examine the association between personality characteristics and scores on self-report measures of driving aggression or recalled driving behaviors. Personality traits that have been shown to relate to aggressive driving tendencies in correlational studies have included dysfunctional impulsivity (Kovácsová et al., 2016); narcissism (e.g., Demir et al., 2016; Lustman et al., 2010; Schreer, 2002); and negative affectivity, trait anger, or hostility (e.g., Beanland et al., 2014; Kovácsová et al., 2016; Sullman et al., 2015). 
One limitation of previous studies that have linked personality traits to driving aggression is the assumption that personality traits are likely to predict behavior reliably across situations. While associations between personality traits and self-reported driving aggression are likely to approximate individuals' driving tendencies in general, a more specific approach that examines the effects of specific motivational states during simulated driving conditions is likely to more closely represent how personality and motivational variables influence actual driving behavior on the road. While there are known limitations to the ecological validity of driving simulation based on factors related to driving simulator design and the behavioral correspondence between simulated driving and actual roadway driving (Deniaud et al., 2015), the use of state-based measures of motivation and personality and physiological measures can help to offset these issues. The present study represents an early attempt to employ driving simulation tasks and state-based personality and motivational measures, in addition to measures of physiological states and personality traits, in order to assess the impact of personality and motivation on driving behavior in an approximation of a real-world context.

A theoretical model that is well suited to such an analysis is reversal theory (Apter, 1982). Reversal theory is a theory of motivation and personality that posits that individuals switch between bi-stable states that represent opposite ways of interpreting motivational experience. For example, experiencing high arousal is desirable when one is in an excitement-seeking, impulsive state, but aversive when one is in a goal-directed state, where high arousal is disruptive and associated with anxiety (Apter, 1976, 1981). Reversal theory focuses on four pairs of states that influence our everyday experiences. These are goal-directed (termed "telic" in reversal theory) vs. arousal-seeking (paratelic), negativistic (i.e., taking pleasure in breaking rules or social conventions) vs. conformist, mastery vs. sympathy, and self- vs. other-oriented states. There is considerable empirical support that individuals experience and switch between these states, and a growing body of research has supported the connection between risk-taking behavior and states posited by reversal theory, including sexual risk-taking (Skakoon-Sparling \& Cramer, 2016), risk-taking associated with substance use (Ianni \& Lafreniere, 2014; O'Connell, et al., 1990), risky sports (Cogan \& Brown, 1999; Kerr \& Mackenzie, 2012), and multiple health-related risks (Kerr et al., 1993; Lafreniere et al., 2013). Most of these studies have found that risky behavior occurs when individuals are in an activity-oriented (paratelic) state in which high arousal is sought. Other research has examined reversal theory constructs in relation to aggressive behavior, including aggression in the context of sports (e.g., Grange \& Kerr, 2011; Rosario et al., 2014) and enjoyment of violence on television (Portell \& Mullet, 2014). To date, no published studies have examined reversal theory's moti- vational states in relation to risky or aggressive driving behaviors.

Reversal theory emphasizes that motivational states at a particular time or in a particular situational context may differ from one's enduring personality traits, and these motivational switches or psychological reversals are of primary interest in the theory (Apter, 1982). The theory is well-suited to examining the motivational frame of mind of individuals prior to and immediately following their behavior in a driving simulator. In addition, the theory suggests that high or low arousal is experienced differently, as either positive or aversive, depending on the person's current motivational state. Some previous research (unrelated to reversal theory) has shown that relative to controls, aggressive drivers show greater physiological reactivity, including elevated systolic blood pressure, when reading driving vignettes (Malta et al., 2001). The present research is a pilot investigation of these relationships in which we examined state-based measures of motivational constructs, negative affectivity, and anger, as well as changes in physiological measures (heart rate and systolic and diastolic blood pressure), in response to driving behaviors in a driving simulator. Findings from this research could increase our knowledge regarding how individuals' motivational state and experience of arousal influence their actual driving behaviors. This information has practical implications for driver education, as well as possible implications for vehicle design and roadway conditions.

\section{The Present Research}

The present study involved two research phases. In Phase 1, student volunteers were recruited to complete an online survey that included measures of personality and metamotivational dominance to examine their associations with propensity for aggressive driving. Our specific focus was to examine relations between telic/paratelic dominance and negativist/conformist dominance and driving aggression, based on previous research that found relations between negativism and engagement in risky behaviors, and because impulsivity was expected to be related to paratelic dominance. In Phase 2, participants who had completed Phase 1 and who agreed to be contacted for the second phase were recruited for a pilot study in which we could observe their simulated driving behavior, as well as examine their pre- and post-test physiological and mood states, to see whether these were influenced by their experience in the driving simulator. The pilot study also served to ensure that equipment and procedures in the driving simulation worked properly in preparation for examining the same effects in a larger study.

In this study, we explored the following research questions: Are paratelic dominance and negativism related to the propensity for aggressive driving? Do these reversal theory constructs account for unique variance in predicting aggressive driving tendencies, beyond what is accounted for by 
other well-established personality predictors of driving aggression? We hypothesized that paratelic dominance and negativism dominance (i.e., rebelliousness) would be associated with propensity for aggressive driving in the psychometric study (phase 1).

The pilot study (phase 2) was preliminary and exploratory and too small in sample size to give rise to specific hypotheses. At the same time, we were interested in participants' metamotivational states before and after their session in the driving simulator, and whether these changed as a result of their simulated driving experience. We also wished to examine whether metamotivational state variables were related to changes in physiological or mood states after the driving simulation.

\section{Phase 1 Method}

\section{Participants}

For Phase 1 we recruited 270 undergraduate student participants from the psychology participant pool of a mediumsized Canadian university. The study was open to participants who were licensed drivers with recent driving experience. The sample was comprised of 212 females (78.5\%), 53 males $(19.6 \%)$, and 5 who listed a gender other than male or female $(1.9 \%)$, with the majority of participants identifying as White/European Canadian (71.5\%). Participants ranged in age from 18 to 68 years $(M=24.39, S D=7.73)$, and reported from 1 to 50 years driving experience $(M=7.23, S D$ = 7.54). The majority of participants reported driving daily or almost daily $(74.4 \%)$, always using seatbelts $(92.2 \%)$, and rated their driving as being "good" or "excellent." Participants' detailed demographic characteristics and driving information are shown in Table 1 . The student volunteers received one bonus point toward their grade in an applicable psychology course in exchange for their participation. The research was cleared by the university's Research Ethics Board and all participants were treated in accordance with the ethical principles of the Canadian Psychological Association and the American Psychological Association.

\section{Procedure and Measures}

Students who were part of the psychology participant pool were able to view an ad for the study online and qualifying participants were given the URL for the online survey. They were first presented with a consent form, and upon agreeing to participate in the study, were taken to the online survey. At the end, participants were asked whether they would be willing to be contacted for possible participation in a further phase of the research investigation. The entire session took less than 30 minutes.

The online survey included the following measures:

The Propensity for Angry Driving Scale (PADS; DePasquale et al., 2001) is a self-report measure that presents
Table 1

Participant Demographic Characteristics and Driving Experiences

\begin{tabular}{|c|c|c|c|c|}
\hline & \multicolumn{2}{|c|}{$\begin{array}{c}\text { Phase } 1 \text { Sample } \\
\quad N=270\end{array}$} & \multicolumn{2}{|c|}{$\begin{array}{l}\text { Pilot Subsample } \\
\qquad N=12\end{array}$} \\
\hline & $n$ & $\%$ & $n$ & $\%$ \\
\hline \multicolumn{5}{|l|}{ Gender } \\
\hline Male & 53 & 19.6 & 1 & 8.3 \\
\hline Female & 212 & 78.5 & 11 & 91.7 \\
\hline Other gender identity & 5 & 1.9 & & \\
\hline \multicolumn{5}{|l|}{ Ethnicity } \\
\hline White/ European/ Canadian & 193 & 71.5 & 8 & 66.7 \\
\hline Middle Eastern & 20 & 7.4 & 1 & 8.3 \\
\hline Black/African/Caribbean & 14 & 5.2 & 1 & 8.3 \\
\hline South Asian & 10 & 3.7 & & \\
\hline Latin/South American & 8 & 3 & 1 & 8.3 \\
\hline East Asian & 6 & 2.2 & & \\
\hline Indigenous & 1 & .4 & & \\
\hline Multiple ethnicities & 13 & 4.8 & & \\
\hline Other or not specified & 5 & 1.8 & 1 & 8.3 \\
\hline \multicolumn{5}{|l|}{ Driving frequency } \\
\hline Rarely/not at all & 3 & 1.1 & & \\
\hline Once or twice per month & 18 & 6.7 & 1 & 8.3 \\
\hline About once per week & 15 & 5.6 & & \\
\hline 2 or 3 times per week & 33 & 12.2 & 2 & 16.7 \\
\hline Daily or nearly daily & 201 & 74.4 & 9 & 75 \\
\hline \multicolumn{5}{|l|}{ Seatbelt use } \\
\hline Never & 1 & .4 & & \\
\hline Almost never & 3 & 1.1 & & \\
\hline Some of the time & 7 & 2.6 & & \\
\hline Most of the time & 10 & 3.7 & & \\
\hline Always & 249 & 92.2 & 12 & 100 \\
\hline \multicolumn{5}{|l|}{ Self-rated driving } \\
\hline Poor & 3 & 1.1 & & \\
\hline Fair & 12 & 4.4 & 1 & 8.3 \\
\hline Average & 57 & 21.1 & 2 & 16.7 \\
\hline Good & 144 & 53.3 & 9 & 75 \\
\hline \multirow[t]{2}{*}{ Excellent } & 54 & 20 & & \\
\hline & $M$ & $S D$ & $M$ & $S D$ \\
\hline Age (years) & 24.39 & 7.73 & 29.17 & 11.38 \\
\hline Years driving & 7.23 & 7.54 & 10 & 10.84 \\
\hline Number of accidents & 0.62 & .84 & 0.67 & .78 \\
\hline $\begin{array}{l}\text { Number of serious accidents } \\
\text { (hospital/injuries) }\end{array}$ & 0.10 & .38 & 0.08 & .29 \\
\hline
\end{tabular}


Table 2

Phase 1 Descriptive Statistics and Intercorrelations for Primary Variables

\begin{tabular}{lrrrrrrr}
\hline Variable & $\alpha$ & \multicolumn{1}{c}{$M$} & \multicolumn{1}{c}{$S D$} & 1 & 2 & 3 & 4 \\
\hline 1. Negativism Dominance & .67 & -7.26 & 5.83 & - & & & \\
2. Telic Dominance & .75 & 3.5 & 4.74 & -.42 & - & & \\
3. Propensity for Angry Driving Scale & .83 & 38.91 & 11.73 & .29 & -.20 & - & \\
4. Impulsivity & .82 & 14.87 & 2.87 & .38 & -.40 & .41 & - \\
5. Narcissism & .87 & 53.91 & 7.07 & .29 & -.23 & .29 & .26 \\
\hline
\end{tabular}

Note. $\alpha=$ Cronbach's alpha. All correlations are significant at $p<.05$.

Table 3

Regression Results for Predictors of Propensity for Aggressive Driving

\begin{tabular}{lccc}
\hline Predictor Variable & $\beta$ & $M$ & $s r^{2}$ \\
\hline Impulsivity & .32 & $5.20 * *$ & .08 \\
Narcissism & .17 & $2.96^{* *}$ & .03 \\
Negativism Dominance & .13 & $2.05 *$ & .01 \\
Telic Dominance & .03 & .42 & .00 \\
\hline Note. $R^{2}=.22 ; * * p<.01, * p<.05$ & &
\end{tabular}

individuals with 19 different driving scenarios and has them select their preferred responses using a multiple-choice format. This measure has been used extensively in psychological research on risky driving, and its reliability, validity, and advantages over other similar measures have been supported in subsequent research (e.g., Dahlen \& Ragan, 2004; Sullman \& Stephens, 2013). The PADS showed strong internal consistency reliability in the present study (Cronbach's alpha $=.83)$.

The Motivational Style Profile (MSP; Apter et al., 1998) consists of 70 items in which responses are completed on a 6-point Likert scale, ranging from 1 (never) to 6 (always). The MSP assesses dominance in the major metamotivational states posited by reversal theory (telic/paratelic, negativistic/conformist, mastery/sympathy, and autic/alloic). A dominance score is derived for each of these pairs of states by subtracting the score for the second state within a pair from the first (e.g., telic dominance is derived from the telic score minus the paratelic score). In the present study, only the telic dominance and negativist dominance subscales were of interest, and their internal consistency was found to be adequate, as shown in Table 2.

The Dysfunctional Impulsivity subscale of the Dickman Impulsivity Inventory (Dickman, 1990) was used to assess impulsivity in the present study. This is a 12-item subscale that asks a series of questions with a yes/no format. The Dickman Impulsivity Inventory has been used in previous studies of aggressive driving (e.g., Kovácsová et al., 2016) and showed good internal consistency reliability (Cronbach's alpha $=.82)$ in the present study.
Narcissism was measured by the Narcissistic Personality Inventory in the present study (NPI; Raskin \& Terry, 1988). The NPI is a 40-item forced-choice inventory that has been widely used in personality research, including research on driving aggression (e.g., Schreer, 2002). The reliability and validity of the NPI have been empirically supported in a number of investigations (e.g., Hasanvand et al., 2015) and the measure showed strong internal consistency reliability (Cronbach's alpha $=.87$ ) in the present investigation.

In addition, participants were asked to provide background information and details about their driving experiences, as shown in Table 1.

\section{Phase 1 Results}

Descriptive statistics and intercorrelations among the primary variables are shown in Table 2. Negativism dominance was positively associated with propensity for aggressive driving, narcissism, and impulsivity, and negatively correlated with telic dominance. Individuals who were high in negativism dominance were also less likely to report using seatbelts consistently $(r=-.22, p<.01)$ and had a greater history of accidents and other negative driving incidents $(r=$ $.16, p<.05)$.

The patterns of negative correlations between telic dominance and the other variables indicate that paratelic dominance was positively associated with propensity for aggressive driving, narcissism, and impulsivity. In addition, paratelic dominance was associated with a lower likelihood of consistent seatbelt use $(r=-.18, p<.01)$.

To determine whether reversal theory constructs added any unique variance to the prediction of aggressive driving beyond the other known predictors of impulsivity and narcissism, a standard multiple regression analysis was conducted with propensity for aggressive driving as the outcome measure. The final regression model is shown in Table 3. The overall regression model was significant, $R^{2}=.22, F(3,262)=17.95, p<.001$. Examination of the squared semi-partial correlation coefficients showed that negativism dominance made a small but significant unique contribution to the prediction of propensity for aggressive driving $\left(\beta=.13, s r^{2}=.01\right)$, although it accounted for considerably less variance than did impulsivity $\left(\beta=.32, s r^{2}=.08\right)$ 
and narcissism $\left(\beta=.17, s r^{2}=.03\right)$. Paratelic dominance (i.e., low telic dominance) did not contribute significantly to the prediction of aggressive driving tendencies in the regression analysis.

\section{Phase 2 Method (Pilot Study)}

\section{Participants}

Twelve participants who had participated in Phase 1 and who indicated a willingness to participate in a further phase of the research comprised the pilot study subsample. Eleven females $(91.7 \%)$ and 1 male $(8.3 \%)$ participated in the pilot study. Their demographic data and driving experience tended to be similar to that of participants in the full Phase 1 sample, except that they were somewhat older on average $(M=29.17$ years, $S D=11.38)$, and consequently tended to have more years of driving experience $(M=10.00$ years, $S D$ $=10.84$ ). A detailed comparison of the subsample to the full Phase 1 sample is shown in Table 1. Pilot participants received one bonus point toward their grade in an applicable psychology course in exchange for their participation. This phase of the research received separate clearance from the university's Research Ethics Board, and all participants were treated in accordance with the ethical principles of the Canadian Psychological Association and the American Psychological Association.

\section{Measures and Equipment}

Phase 2 involved pre- and post-test administration of psychometric and physiological measures and completion of the driving simulation. Each of these methods of assessment is described below.

The Positive and Negative Affect Schedule (PANAS; Watson et al., 1988) was administered at pre- and post-test to assess current positive and negative mood states. The PANAS consists of 20 words representing emotions and mood states that participants rate on a 5-point rating scale from "very slightly or not at all" to "extremely". This measure has been used extensively in research on mood and negative affectivity, including in previous research on driving behaviors (e.g., Britt \& Garrity, 2006; Parkinson, 2001).

The State Anger subscale of the State-Trait Anger Expression Inventory-2 (STAXI-2; Spielberger, 1999) was used to assess pre- and post-test anger states. The State Anger subscale consists of 15 items related to expressions of current anger that are rated on a 4-point scale (from "not at all" to "very much so"). The current and previous versions of the STAXI-2 have shown robust reliability and validity in previous studies, including studies related to driving aggression (e.g., Malta et al., 2001).

A modified version of the State of Mind Indicator for Athletes (SOMIFA; Kerr \& Apter, 1999) was used to measure current metamotivational states. The measure was adapted to assess how participants felt at pre-test and while they were driving in the simulator. The SOMIFA assesses all metamotivational states specified by reversal theory in a brief 11-item measure.

Physiological measures. Systolic and diastolic blood pressure (BP) and heart rate were assessed at baseline, preand post-test through an Omron 10 portable blood pressure monitor. Participants were instructed in the use of the BP monitor by a research assistant (RA). Participants placed the cuff on themselves and the RA pressed start on the monitor to begin the electronic recording of $\mathrm{BP}$ and heart rate. A sticky note was placed over the monitor's screen to shield the readings from the participant, in order to prevent reactivity that might influence subsequent readings. The RA noted the readings and would only provide feedback to participants at the end of the experimental session if participants requested it or if the readings were abnormally out of range and warranted discontinuation of the session. No concerning out-of-range values were observed in the pilot study.

Driving simulation. Participants' driving behavior was observed as they completed scenarios that had been created using a MiniSim driving simulator from National Advanced Driving Simulator (NADS). The simulator resembles the driver's seat of an automobile, equipped with a steering wheel and dashboard, gas and brake pedals, turning signals on the steering column, etc. Three large screens placed in front of the driver's seat present an interactive visual display of various challenging driving scenarios. There were nine driving scenarios that were designed to provoke aggressive driving, including being forced to drive behind a slow-moving truck, anticipation of lane restrictions due to a construction zone, an unreasonably slow speed limit, and suddenly being cut-off by another vehicle. The simulator records various driving parameters, including driving speed, lane changes, accelerator and brake pedal force, vehicle following distance, and horn use.

\section{Procedure}

Participants entered the lab and were greeted by a Psychology RA who briefly explained the procedures and administered a consent form. After the participant signed the consent form, the RA took and recorded their baseline levels of BP and heart rate. Next, participants were guided to a computer where they completed the pre-test psychometric measures in the following order: PANAS, State Anger subscale, and the modified SOMIFA. Following their completion of these measures, the RA took pre-test readings of their BP and heart rate.

At this point, the Psychology RA took the participant to another part of the lab where an RA from Civil and Environmental Engineering oriented the participant to the driving simulator. The participant was asked to test drive the simulator for two to three minutes in order to become comfortable 
with its operation. The participant was told to inform the Engineering RA if they experienced any dizziness or other symptoms of motion sickness at any point during the driving simulation. Following the brief test drive, the participant drove the simulator through the nine traffic scenarios with the Engineering RA nearby.

Upon completion of the driving scenarios, the participant returned to the Psychology RA, who immediately took posttest readings of BP and heart rate. Following the physiological measures, the participant completed the psychometric measures in the following order: modified SOMIFA, State Anger subscale, and PANAS. This order allowed us to administer the SOMIFA right after the post-test physiological measures, in an attempt to determine post-driving metamotivational states before participants completed further questionnaire measures that might alter their states. When the participant had completed these measures on the computer, the Psychology RA indicated that the experimental procedures were complete, answered any questions that the participant had, and confirmed the arrangements for participant compensation (the awarding of 1 bonus point through the online Participant Pool system). The participant was then thanked and dismissed from the session. The entire experimental session took approximately one hour to complete.

\section{Phase 2 Results (Pilot Study)}

Pilot study results are based on a small sample of 12 participants, so all findings should be treated with some caution and regarded as worthy of further exploration in a larger sample. Participants in the pilot study subsample were similar to those in the large sample from which they were recruited in most background and driving characteristics, but were older on average and had more driving experience, as can be seen in Table 1.

The modified SOMIFA allowed us to assess the metamotivational state of the participants before and after engaging in the driving simulation. Most participants remained in the same state at pre- and post-test. Five participants started out in the telic state and remained in that state, and four participants were in the paratelic state at both pre- and post-test. Three participants showed a state reversal, and in all three cases, they switched from the telic to the paratelic state after experiencing the driving simulation. The majority of participants (11) were in the conformist state before the driving session and remained in that state, while one participant was in the negativistic state at both pre- and post-test.

Phase 2 participants were categorized as being high or low in negativism, based on a median split of their Phase 1 MSP negativist dominance scores, and classified as high or low on propensity for aggressive driving, based on a median split of their Phase 1 scores on the PADS. Individuals who were high in negativism were significantly more likely to be high in propensity for aggressive driving, and low negativism cor-

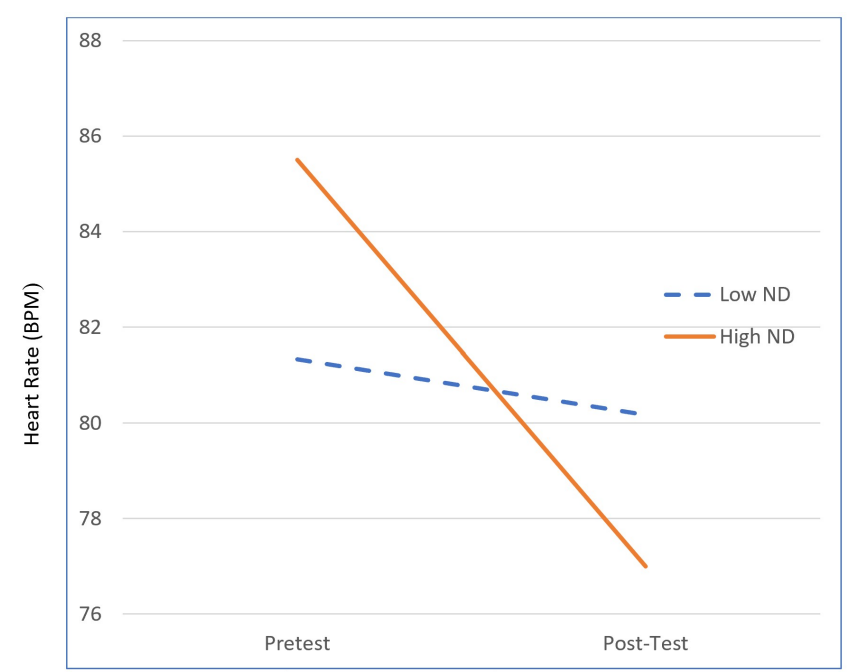

Figure 1. Pre- and Post-Test Heart Rate for Individuals High and Low in Negativism Dominance (ND)

responded to lower propensity for aggressive driving, $\chi^{2}(1)$ $=5.33, p=.021$.

A series of repeated-measures ANOVAs was conducted to examine whether participants' physiological indicators and mood states changed from pre-test to post-test, and whether these changes interacted with negativism. Given the very small sample size, a significance level of .10 was adopted to further interpret results (Field, 2020).

Pre- and post- driving levels of $\mathrm{BP}$ and heart rate were examined. There were no significant effects for systolic or diastolic blood pressure, but analyses of pre- and post-test levels of heart rate yielded some significant effects. Findings showed a main effect for Time, in which heart rate decreased after the completion of the driving simulation, $F(1,10)=$ $7.91, p=.018$. There was also a significant Time $\mathrm{x}$ Negativism Group interaction, $F(1,10)=4.55, p=.059$. Individuals who were high in negativism showed a greater reduction in heart rate after the driving simulation, compared to those low in negativism. This interaction is illustrated in Figure 1.

Changes in mood states were also examined. There were no significant effects of positive affect, but negative affect showed an interesting Time $x$ Negativism Group interaction. For individuals who were low in negativism, negative affect increased after the simulated driving session, while for those high in negativism, the opposite pattern was shown, in which their negative affect decreased at post-test, $F(1,10)=$ $3.50, p=.091$, as shown in Figure 2. A similar trend (albeit not quite statistically significant) was seen for state anger, in which more negativistic individuals showed a reduction in anger after the driving simulation, while those low in negativism showed increased anger at post-test, $F(1,10)=$ $2.69, p=.132$. 


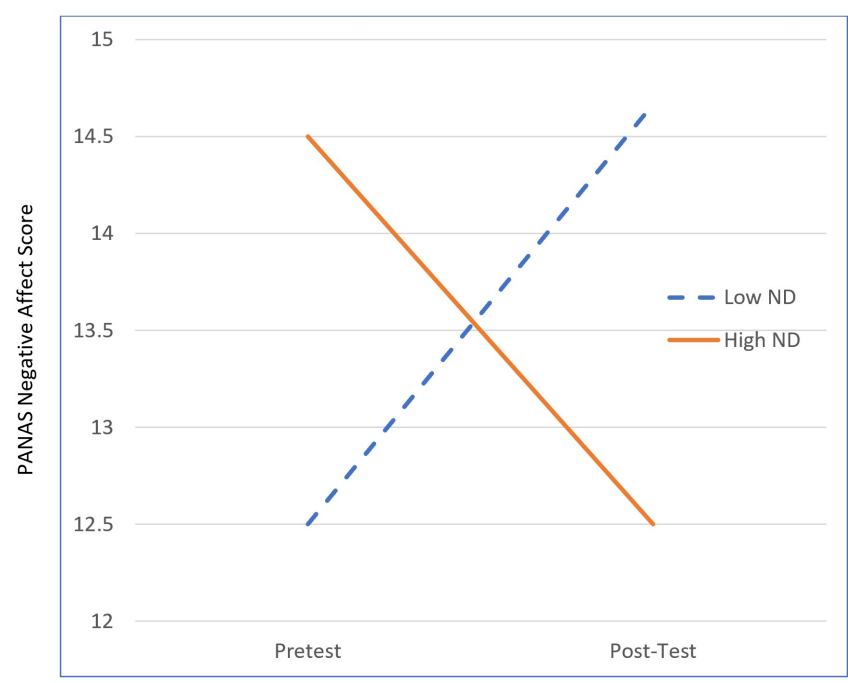

Figure 2. Pre- and Post-Test Negative Mood State for Individuals High and Low in Negativism Dominance (ND)

A limited amount of driving data was extracted from the simulator that allowed us to observe the simulated driving behavior of a few participants. We selected two participants who differed in their metamotivational dominance to compare on a few specific driving parameters. These participants are described, using pseudonyms, in Table 4.

Recorded driving responses by Hannah and Kelly to two driving scenarios are shown in Figure 3. In the first scenario, shown at the top, the driver approaches an intersection just as the light turns red. While aggressive drivers may run through the red light, both Hannah and Kelly stopped at the intersection. The differences in brake pedal force are depicted in Figure 3, in which Kelly jammed on the brake very hard, while Hannah braked with considerably less force. In the second scenario, shown at the bottom, the drivers are travelling on a segment of roadway with a lower posted speed limit (60 kmph to $50 \mathrm{kmph}$, then back to $60 \mathrm{kmph}$ ) and no driver ahead of them. Aggressive drivers are likely to exceed the speed limit in this scenario. While Hannah's average driving speed was close to the speed limit of $50 \mathrm{~km} / \mathrm{hr}$, Kelly's average speed was more than $10 \mathrm{~km} / \mathrm{hr}$ over the speed limit and she sometimes reached a speed that exceeded the speed limit by $30 \mathrm{~km} / \mathrm{hr}$. The overall pattern showed stronger indicators of aggressive driving for Kelly, who was negativist dominant and in the paratelic state, as compared to Hannah, who was conformist dominant and in the telic, conformist state combination. While these findings are preliminary and only based on a few driving scenarios for two participants, they illustrate the potential to observe metamotivational influences on aggressive driving in a simulated driving session.
Table 4

Comparison of two specific driving simulator participants

\begin{tabular}{lll}
\hline Participant & "Hannah" & "Kelly" \\
\hline Age & 31 years & 27 years \\
Driving experience & 14 years & 1 year \\
Dominance & $\begin{array}{l}\text { Telic dominant } \\
\text { Conformist dominant }\end{array}$ & $\begin{array}{l}\text { Paratelic dominant } \\
\text { Negativist dominant }\end{array}$ \\
& Telic & Paratelic \\
Pre-test states & Conformist & Conformist \\
& Telic & Paratelic \\
Post-test states & Conformist & Conformist
\end{tabular}

\section{Discussion}

Our findings provide partial support for our hypothesis and demonstrate the utility of examining reversal theory constructs in a driving simulation laboratory experiment. Correlational and regression findings from Phase 1 supported our hypothesis that paratelic dominance and negativism dominance would be positively related to propensity for aggressive driving. In a standard multiple regression analysis that also included personality factors that are well established predictors of propensity for driving aggression (narcissism and impulsivity), negativism dominance emerged as a significant predictor and contributed unique variance. Negativism dominance was also associated with other negative driving behaviors, including using seatbelts inconsistently and having a more extensive history of accidents and other negative driving incidents (e.g., "near-misses" and inattentive driving).

Paratelic dominance (i.e., low telic dominance) was correlated with propensity for aggressive driving but did not emerge as a significant predictor in the regression analysis. At the same time, the significant negative correlation between telic dominance and propensity for driving aggression suggests that this variable should continue to be explored in future research. Findings from Phase 1 showed dysfunctional impulsivity to be a particularly strong predictor of propensity for aggressive driving, followed by narcissism and negativism dominance. These findings are consistent with previous research that has shown impulsivity and narcissism to predict aggressive driving tendencies (Demir et al., 2016; Kovácsová et al., 2016; Lustman et al., 2010; Schreer, 2002). Both paratelic dominance and negativism dominance were correlated with narcissism and impulsivity in the present study, with particularly strong associations with impulsivity. The finding of a negative correlation between telic dominance and impulsivity is consistent with previous research by our team (Lafreniere \& Cramer, 2006) that 


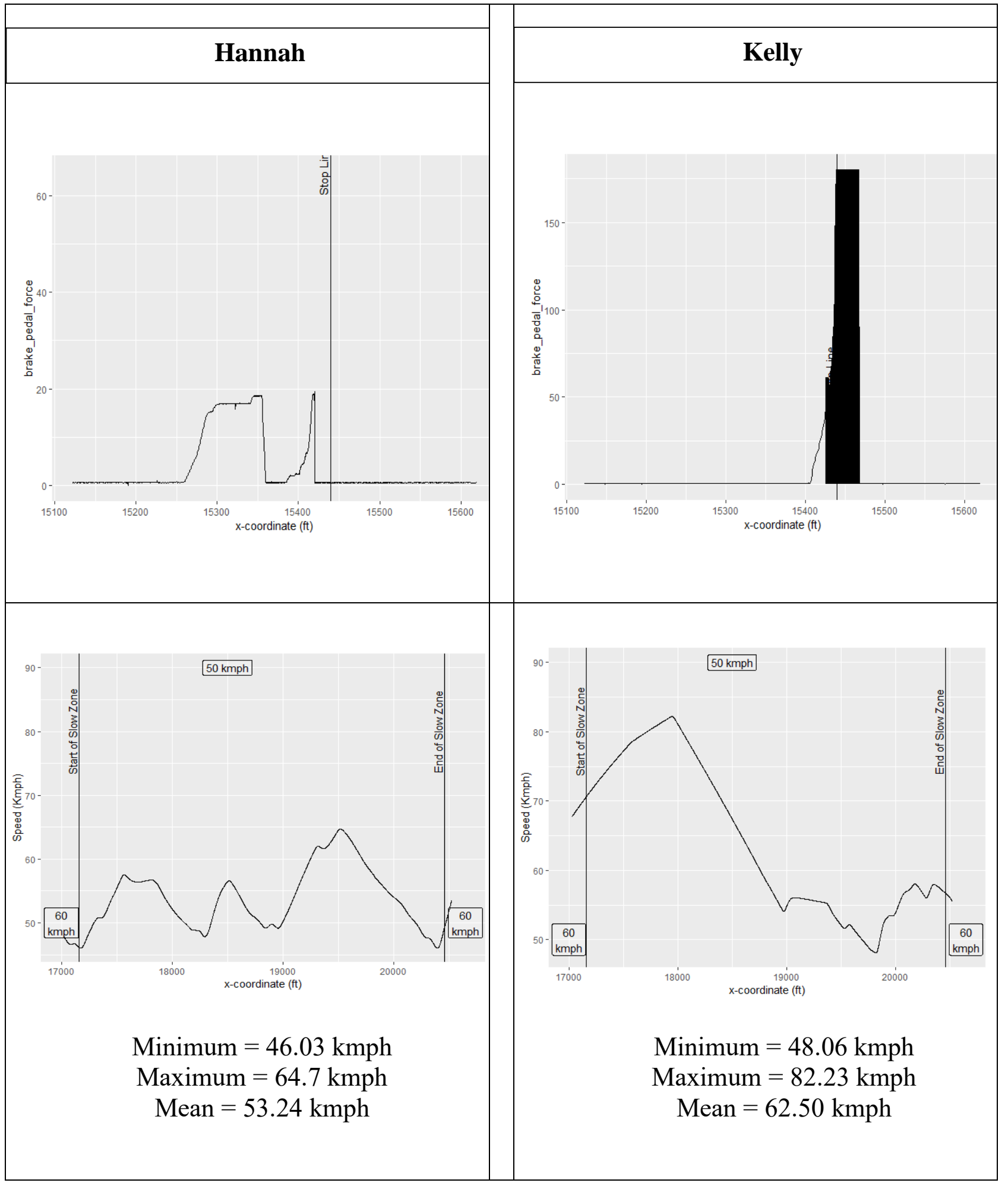

Figure 3. Driving Behavior of a Conformist Dominant (on Left) and a Negativist Dominant Driver (on Right) When Braking for a Red Light and Driving in a Slow Zone 
found that telic dominance was significantly related to the construct "consideration of future consequences" (Strathman et al., 1994), which tends to be negatively related to impulsivity (e.g., Bénard et al., 2018). Consideration of future consequences has also been shown to be negatively related to aggressive driving in previous research (Moore \& Dahlen, 2008). Thus, paratelic dominance, which is negatively associated with consideration of future consequences and positively related to impulsivity, would seem to be a variable worth continuing to examine in risky driving research.

The pilot study was based on a small sample of 12 participants and caution should be exercised when interpreting its findings. At the same time, some interesting patterns emerged that should be explored in future research. The study allowed us to assess metamotivational state at two time points, just before and just after participation in the driving simulation. Most participants (8 out of 12) started out in a telic state before engaging in the driving simulation, while four were in a paratelic state at pre-test. Nine participants remained in their pre-test state, but three participants showed a reversal, and in all cases, they reversed to the paratelic state after the driving simulation. Shifting from the telic to the paratelic state might simply reflect a sense of relief that the biggest part of the experiment was behind them, but examining such shifts in relation to the actual driving behavior displayed in the simulator would yield interesting results in future research.

The majority of participants (11 out of 12) were in the conformist state at pre-test and remained in that state at posttest. One participant was in the negativistic state at both preand post-test. No participants showed conformity-negativism reversals. Participants were in a highly structured laboratory session and had undergone previous experimental procedures, including online questionnaires and physiological readings, which required that they follow the rules and protocols as explained to them. They were also in relatively close proximity to research assistants while they engaged in the driving simulation and they knew that their driving responses were being electronically recorded. This context likely facilitates a conformist state more than it does a negativistic one. This also points out some of the limits to the ecological validity of simulated driving. It is possible that drivers express greater caution and conformity while driving in the simulator than they would on the roadway where they might not feel like they are being immediately monitored, especially in situations where they are driving in sparsely populated areas or late at night. On the other hand, participants know that they are encapsulated from real-world consequences of their driving mistakes in the simulator. It is possible for some participants to treat their simulated driving like a game, since there is no real physical consequence of crashing the car, for example. Whereas the 12 participants that we observed in the pilot study generally showed greater conformity to driving rules and demands of the roadway, it is possible that some participants in the negativistic state would enjoy being as reckless as possible in the simulator.

Participants who scored high on negativism dominance in the pilot sample also tended to score high on propensity for aggressive driving, and a chi-square analysis confirmed that this relationship was statistically significant. This result supports the correlational findings shown in Phase 1 and suggests that negativism predicted aggressive driving tendency in the pilot study. When negativism dominance was examined as a between-subjects factor in a series of repeatedmeasures analyses of variance, results showed that negativist dominant individuals showed significant reductions in negative affect and physiological arousal (as measured by heart rate) after the driving simulation. Conformist dominant participants showed a significantly lower decline in heart rate after the simulated driving, and their negative affect actually increased, in a pattern opposite to those who were negativist dominant. Taken together, these findings suggest that engagement in aggressive driving seems to be more aversive for conformist dominant individuals, in that it leads to increases in negative mood state. Conversely, engaging in challenging driving scenarios led negativist dominant individuals to display reduced signs of tension (i.e., lower heart rate and reduced negative affect). This is an interesting finding, and one might speculate that negativist dominant individuals might be using the driving challenges to work out their feelings of tension and achieve a more relaxed state. If this is the case, it might have concerning implications in a real-life context, since negativist dominant individuals are more prone to anger and dysfunctional impulsivity, and this could lead them to engage in reckless driving as a way to calm themselves down from the stresses and hassles of daily life.

Our future research will allow us to examine the actual driving data from negativistic and conformist participants, to see if negativistic individuals display more risky driving behaviors. A small snapshot of specific driving behaviors of two drivers was examined to look at such differences. One participant (Hannah) was conformist dominant and telic dominant, while the other (Kelly) was negativist dominant and paratelic dominant. Both participants were in the conformist state, but Hannah was in the telic state, while Kelly was in the paratelic state (based on pre- and post-test state measures). The two participants showed marked differences in some driving behaviors, with Hannah showing more tightly controlled driving speed and more careful braking. Kelly, who was negativist dominant and in the paratelic state while driving, showed variable driving speed that went way above the posted speed limit, and an extreme use of the brakes in response to a red light. It is important to note that Kelly had considerably less driving experience than was true for Hannah, and this driving inexperience might account for some of the erratic nature of Kelly's driving. Examination of 
driving profiles of negativistic versus conformist individuals in future samples will help to determine if there are any robust differences in simulated driving that are associated with metamotivational dominance and current metamotivational state.

\section{Implications and Future Research Directions}

Findings from this investigation suggest that tendencies toward aggressive driving are related to negativism and paratelic dominance, and that negativist dominant individuals may respond to challenging driving situations differently than those who are conformist dominant. While our pilot study findings are based on a small sample size, they illustrate the value of examining metamotivational dominance and state in relation to driving behavior. Some limitations of the present research relate to the nature of the participants. In both studies, the participants were student volunteers drawn from a psychology participant pool, and the sample was comprised of a majority of women. Future studies should examine more gender-balanced and community samples, to determine the generalizability of findings.

Since negativism emerged as an important predictor in the present research, it would be interesting to adopt a more nuanced approach to its measurement in future studies. One way would be to use the Rebelliousness Questionnaire (McDermott, 1988) to measure proactive and reactive dimensions of negativism. This would allow us to see whether aggressive drivers are pushing back against the rules and restrictions on the road (reactive rebelliousness) or engaging in provocative, thrill-seeking behavior through reckless driving (proactive rebelliousness). It would also be interesting to extend this research to other areas of dysfunctional driving, including distracted driving and driving while impaired, to see if rebelliousness and its subtypes predict these behaviors as well.

Findings from the present study, if supported in our future research with larger and more diverse samples, have implications for considering personality and metamotivational factors as important influences on driving behavior. These findings might lead to recommendations for driving instruction, in which student drivers could learn to assess their motivational tendencies before going on the road and learn to manage negative mood states in safe ways before they drive. There is also a potential for the development of apps that allow individuals to quickly assess their motivational tendencies and how these might influence their driving behavior. Reversal theory's emphasis on considering the motivational needs that underlie everyday behavior seems ideally suited to such efforts.

\section{References}

AAA Foundation for Traffic Safety. (2016). Prevalence of self-reported aggressive driving behavior:
United States, 2014. AAA Foundation for Traffic Safety. https://aaafoundation.org/prevalence-selfreported-aggressive-driving-behavior-united-states-2014/

Apter, M. J. (1976). Some data inconsistent with the optimal arousal theory of motivation. Perceptual and Motor Skills, 43(3), 1209-1210. https://doi.org/10.2466/pms.1976.43.3f.1209

Apter, M. J. (1981). Reversal theory: Making sense of felt arousal. New Forum, 8, 27-30.

Apter, M. J. (1982). The experience of motivation: The theory of psychological reversals. New York: Academic Press.

Apter, M. J., Mallows, R., \& Williams, S. (1998). The development of the Motivational Style Profile. Personality and Individual Differences, 24, 7-18. https://doi.org/10.1016/S0191-8869(97)00148-7

Beanland, V., Sellbom, M., \& Johnson, A., K. (2014). Personality domains and traits that predict self-reported aberrant driving behaviours in a southeastern US university sample. Accident Analysis and Prevention, 72, 184-192. http://dx.doi.org/10.1016/j.aap.2014.06.023

Bénard, M., Bellisle, F., Etile, F., Reach, G., Kesse-Guyot, E., Hercberg, S., Peneau, S. (2018). Impulsivity and consideration of future consequences as moderators of the association between emotional eating and body weight status. International Journal of Behavioral Nutrition $\mathcal{F}$ Physical Activity 15, 84. https://doi.org/10.1186/s12966-0180721-1

Britt, T. W., \& Garrity, M. J. (2006). Attributions and personality as predictors of the road rage response. British Journal of Social Psychology, 45,127-147. doi:10.1348/014466605X41355

Cogan, N., \& Brown, R. I. F. (1999). Metamotivational dominance, states, and injuries in risk and safe sports. Personality and Individual Differences, 27(3), 503-518. https://doi.org/10.1016/S0191-8869(98)00259-1

Dahlen, E. R., \& Ragan, K. M. (2004). Validation of the Propensity for Angry Driving Scale. Journal of Safety Research, 35, 557-563. doi:10.1016/j.jsr.2004.09.002

Demir, B., Demir, S., \& özkan, T. (2016). A contextual model of driving anger: A meta-analysis. Transportation Research Part F, 42, 332-349. http://dx.doi.org/10.1016/j.trf.2016.09.020

Deniaud, C., Honnet, Jeanne, B., \& Mestre, D. (2015). The concept of "presence" as a measure of ecological validity in driving simulators. Journal of Interaction Science, 3(1), 1-13. https://doi.org/10.1186/s40166-015-0005-z

DePasquale, J. P., Geller, E. S., Clarke, S. W., \& Littleton, L. C. (2001). Measuring road rage: Development of the Propensity for Angry Driving Scale. Journal of Safety Research, 32, 1-16. https://doi.org/10.1016/S00224375(00)00050-5 
Dickman, S. J. (1990). Functional and dysfunctional impulsivity: Personality and cognitive correlates. Journal of Personality and Social Psychology, 58(1), 95-102. doi:10.1037/0022-3514.58.1.95

Field, A. (2020). Discovering statistics using IBM SPSS statistics (5th ed.). London: Sage.

Grange, P., \& Kerr, J. H. (2011). Do elite athletes renowned for their aggressive play transfer aggression to nonsport settings? A qualitative exploratory study. Journal of Aggression, Maltreatment $\mathcal{F}$ Trauma, 20(4), 359-375. http://dx.doi.org/10.1080/10926771.2011.568996

Hasanvand, N. Z., Javanmard, K., \& Goodarzi, M. (2015). Validation of the Narcissistic Personality Inventory-40. Journal of Psychology, 19(1), 102-118.

Hennessey, D. A., \& Wiesenthal, D. L. (2002). The relationship between driver aggression, violence, and vengeance. Violence and Victims, 17(6), 707-718. DOI: 10.1891/vivi.17.6.707.33719

Ianni, P. A., \& Lafreniere, K. D. (2014). Personality and motivational correlates of energy drink consumption and misuse among female undergraduate students. Personality and Individual Differences, 69, 110-114. http://dx.doi.org/10.1016/j.paid.2014.05.022

Kerr, J. H., \& Apter, M. J. (1999). The State of Mind Indicator for Athletes. In J. H. Kerr (Ed.), Experiencing sport: Reversal theory (pp. 239-244). Chichester, England: Wiley.

Kerr, J. H., Frank-Ragan, E., \& Brown, R. I. F. (1993). Taking risks with health. Patient Education and Counseling, 22(2), 73-80. https://doi.org/10.1016/07383991(93)90003-F

Kerr, J. H., \& Mackenzie, S. H. (2012). Multiple motives for participating in adventure sports. Psychology of Sport and Exercise, 13(5), 649-657. https://doi.org/10.1016/j.psychsport.2012.04.002

Kovácsová, N., Lajunen, T., \& Rošková, E. (2016). Aggression on the road: Relationships between dysfunctional impulsivity, forgiveness, negative emotions, and aggressive driving. Transportation Research Part F: Traffic Psychology and Behaviour, 42, 286-298 https://doi.org/10.1016/j.trf.2016.02.010

Lafreniere, K. D., \& Cramer, K. M. (2006). Examining reversal theory measures in relation to NEO personality dimensions and consideration of future consequences. Personality and Individual Differences, 40(7), 1387-1397. doi: 10.1016/j.paid.2005.11.019

Lafreniere, K. D., Menna, R., \& Cramer, K. M. (2013). Rebelliousness, effortful control, and risky behavior: Metamotivational and temperamental predictors of risk-taking in older adolescents. Journal of Motivation, Emotion, and Personality, 1(1), 17-26. doi: 10.12689/jmep.2013.103

Lustman, M., Wiesenthal, D.L., \& Flett, G.L. (2010). Narcissism and aggressive driving: Is an in- flated view of the self a road hazard? Journal of Applied Social Psychology, 40(6), 1423-1449. https://doi-org.ledproxy2.uwindsor.ca/10.1111/j.15591816.2010.00624.x

Malta, L. S., Blanchard, E. B., Freidenberg, B. M., Galovski, T. E., Karl, A., \& Holzapfel, S. R. (2001). Psychophysiological reactivity of aggressive drivers: An exploratory study. Applied Psychophysiology and Biofeedback, 26(2), 95-116. https://doi.org/10.1023/A:1011373105966

McDermott, M. R. (1988). Measuring rebelliousness: The development of the Negativism Dominance Scale. In M. J. Apter, J. H. Kerr, \& M. P. Cowles (Eds.), Progress in reversal theory (pp. 297-312). Amsterdam: Elsevier.

McTish, P., \& Park, S. (2016). Exploring aggressive driving behavior in Pennsylvania's Delaware Valley region. Proceeding of International Conference on Sustainable Design, Engineering and Construction.

Moore, M., \& Dahlen, E. R. (2008). Forgiveness and consideration of future consequences in aggressive driving. Accident Analysis and Prevention, 40, 1661-1666. https://doi.org/10.1016/j.aap.2008.05.007

O’Connell, K. A., Cook, M.R., Gerkovich, M. M., Potocky, M., \& Swan, G. E. (1990). Reversal theory and smoking: A state-based approach to ex-smokers' highly tempting situations. Journal of Consulting and Clinical Psychology, 58(4), 489-494. doi: 10.1037//0022-006x.58.4.489.

Parkinson, B. (2001). Anger on and off the road. British Journal of Psychology, 92, 507-526.

Portell, M., \& Mullet, E. (2014). Why do people enjoy watching natural disasters and human violence on television? A reversal theory perspective. Journal of Motivation, Emotion, and Personality, 2(1), 38-49. doi: 10.12689/jmep.2014.205

Raskin, R., \& Terry, H. (1988). A principal-components analysis of the Narcissistic Personality Inventory and further evidence of its construct validity. Journal of Personality and Social Psychology, 54(5), 890-902.

Rosario, D., Kerr, J. H., \& Rhodius, A. (2014). The experience of aggression among mixed martial arts athletes interpreted through reversal theory. International Journal of Sport Psychology, 45(2), 79-99. doi:10.7352/IJSP.2014.45.079

Schreer, G. (2002). Narcissism and aggression: Is inflated self-esteem related to aggressive driving? North American Journal of Psychology, 4(3), 333-342.

Skakoon-Sparling, S., \& Cramer, K. M. (2016). The impact of sexual arousal on elements of sexual decision making: Sexual self-restraint, motivational state, and self-control. Canadian Journal of Human Sexuality, 25(2), 119-125. doi: 10.3138/cjhs.252-A1

Spielberger, C. D. (1999). State-Trait Anger Expression Inventory-2 (STAXI-2). Professional manual. Tampa, FL: Psychological Assessment Resources. 
Strathman, A., Gleicher, F., Boninger, D. S., \& Edwards, C. S. (1994). The consideration of future consequences: Weighing immediate and distant outcomes of behavior. Journal of Personality and Social Psychology, 66(4), 742752. https://doi.org/10.1037/0022-3514.66.4.742

Sullman, M. J. M., \& Stephens, A. N. (2013). A comparison of the Driving Anger Scale and the Propensity for Angry Driving Scale. Accident Analysis and Prevention, 58, 8896. https://doi.org/10.1016/j.aap.2013.05.002

Sullman, M. J. M., Stephens, A. N., \& Yong, M. (2015). Anger, aggression and road rage behaviour in Malaysian drivers. Transportation Research Part F: Traffic Psychology and Behaviour, 29, 70-82. https://doi.org/10.1016/j.trf.2015.01.006

Traffic Injury Research Foundation (n.d.). The road safety monitor - speeding and aggressive driving. https://tirf.ca/projects/road-safety-monitor-speedingaggressive-driving/

Watson, D., Clark, L. A., \& Tellegen, A. (1988). Development and validation of brief measures of positive and negative affect. The PANAS scales. Journal of Personality and Social Psychology, 54(6), 1063-1070. 